\title{
Massa corporal e estatura autorreferidas para avaliação do estado nutricional de estudantes universitários
}

\author{
Roberto Fernandes da Costa ${ }^{1}$ \\ 'Escola de Saúde e Bem-Estar, Faculdade de Desenvolvimento do Rio Grande do Sul (FADERGS) - Porto Alegre (RS), Brasil. E-Mail: roberto.costa @ fadergs.edu.br
}

DOI: http://dx.doi.org/10.7322/abcshs.v41i2.870

A condução de estudos epidemiológicos do estado nutricional envolvendo grandes grupos populacionais nem sempre permite a realização de medidas antropométricas in loco, pois mesmo as mais simples - como massa corporal e estatura - dependem de recursos materiais e humanos para a sua realização, além das medidas antropométricas aumentarem o risco de recusa ${ }^{1,2}$. Nesse sentido, tem sido frequente a utilização de medidas autorreferidas de massa corporal e estatura para a estimativa do estado nutricional pelo índice de massa corporal (IMC) $)^{3-6}$.

Apesar da praticidade decorrente do uso de medidas autorreferidas, ainda são conflitantes os resultados quanto à sua validade $\mathrm{e}^{7,8} \mathrm{e}$ escassos os estudos com universitários em nosso país².

Esta edição da ABCS Health Sciences traz dois artigos sobre o tema, contribuindo substancialmente para o conhecimento na área. O estudo de Souza e Barbosa ${ }^{9}$ testou a validade de medidas autorreferidas de massa corporal e estatura em estudantes universitários do estado da Bahia, verificando que, embora a maior faixa etária dos participantes e o maior tempo de exposição à vida universitária possam ser fatores associados ao erro entre medidas referidas e aferidas, os níveis de concordância foram satisfatórios, caracterizando boa aplicabilidade dessa alternativa para estudos de tal natureza.

Já o estudo de Rechenchosky et al. ${ }^{10}$ analisou a concordância entre valores de massa corporal e estatura autorreferidos e medidos para o diagnóstico do estado nutricional de estudantes universitários de Educação Física do estado de Goiás. Os autores verificaram que, de modo geral, a obtenção dessas medidas, bem como sua utilização para a avaliação do estado nutricional, demonstrou validade suficiente, constituindo uma boa alternativa para esse tipo de levantamento. Cabe ressaltar que, na estratificação pelo estado nutricional, o grupo formado por sujeitos com obesidade apresentou concordância abaixo do mínimo necessário.

Ambos os estudos utilizaram técnicas estatísticas robustas para a comparação e a verificação da concordância entre valores autorreferidos e medidos, incluindo, além dos testes de comparação de médias ou medianas, o coeficiente de correlação intraclasse (CCI), o teste de Kappa e a plotagem de Bland-Altman, o que confere bom poder de inferência aos resultados obtidos.

Embora alguns estudos recentes venham tentando demonstrar os riscos de viés do uso de medidas autorreferidas ${ }^{5,7}$, este continua sendo uma alternativa viável, válida e econômica para grandes levantamentos de dados antropométricos e para a investigação do estado nutricional ${ }^{1,4,6}$.

A associação do erro com a maior faixa etária observada no estudo de Souza e Barbosa ${ }^{9}$ a baixa concordância encontrada em sujeitos com obesidade no estudo de Rechenchosky et al..$^{10}$ estão em concordância com achados da literatura internacional ${ }^{3,8}$. Para a população universitária da área da saúde, especialmente, Pregnolato et al. ${ }^{2}$ verificaram validade para a avaliação do estado nutricional por medidas autorreferidas apenas para o sexo masculino, porém não empregaram técnicas estatísticas tão refinadas quanto às dos artigos publicados nesta edição da ABCS Health Sciences.

Considerando que um dos estudos contou com amostra de estudantes universitários de vários cursos ${ }^{9} \mathrm{e} o$ outro apenas com acadêmicos de Educação Física ${ }^{10}$, além das particularidades de cada estudo deles quanto à abordagem e à discussão dos resultados obtidos, a análise conjunta dos artigos permitirá ao leitor um melhor entendimento sobre o tema e a ampliação dos argumentos para a utilização de medidas autorreferidas de massa corporal e estatura para a avaliação do estado nutricional em estudos epidemiológicos. 


\section{REFERÊNCIAS}

1. Niedzwiedzka E, Dlugosz A, Wadolowska L. Validity of selfreported height and weight in elderly Poles. Nutr Res Pract. 2015;9(3):319-27.

http://dx.doi.org/10.4162/nrp.2015.9.3.319

2. Pregnolato TS, Mesquita LM, Ferreira PG, Santos MM, Santos CC, Costa RF. Validade de medidas autorreferidas de massa e estatura e seu impacto na estimativa do estado nutricional pelo índice de massa corporal. Rev Bras Cresc Desenvolv Hum. 2009;19(1):35-41. http://dx.doi.org/10.7322/jhgd.19900

3. Merrill RM, Richardson JS. Validity of self-reported height, weight, and body mass index: findings from the National Health and Nutrition Examination Survey, 2001-2006. Prev Chronic Dis. 2009;6(4):A121.

4. Neovius K, Johansson K, Kark M, Tynelius P, Rasmussen F. Trends in self-reported BMl and prevalence of obesity 2002-10 in Stockholm County, Sweden. Eur J Public Health. 2013;23(2):312-5. http://dx.doi.org/10.1093/eurpub/cks128

5. Stommel M, Osier N. Temporal changes in bias of body mass index scores based on self-reported height and weight. Int $\mathrm{J}$ Obes (Lond). 2013;37(3):461-7. http://dx.doi.org/10.1038/ijo.2012.67

6. Aasvee K, Rasmussen M, Kelly C, Kurvinen E, Giacchi MV, Ahluwalia N. Validity of self-reported height and weight for estimating prevalence of overweight among Estonian adolescents: the Health Behaviour in School-aged Children study. BMC Res Notes. 2015;8:606.

http://dx.doi.org/10.1186/s13104-015-1587-9
7. Ikeda N. Validity of self-reports of height and weight among the general adult population in Japan: findings from National Household Surveys, 1986. PLoS One. 2016;11(2):e0148297. http://dx.doi.org/10.1371/journal.pone.0148297

8. Lim LL, Seubsman SA, Sleigh A. Validity of self-reported weight, height, and body mass index among university students in Thailand: implications for population studies of obesity in developing countries. Popul Health Metr. 2009;7:15. http://dx.doi.org/10.1186/1478-7954-7-15

9. Sousa TF, Barbosa AR. Validade das medidas referidas da massa corporal e estatura em universitários. ABCS Health Sci. 2016:41(2):71-77.

http://dx.doi.org/10.7322/abcshs.v41i2.872

10. Rechenchosky L, Ines LL, Oliveira LAS. Concordância entre valores autorreferidos e mensurados de massa corporal e estatura para o diagnóstico do estado nutricional em universitários de Educação Física. ABCS Health Sci. 2016;41(2):63-70.

http://dx.doi.org/10.7322/abcshs.v41i2.871 